Proc. Indian Acad. Sci. (Earth Planet. Sci.), Vol. 101, No. 4, December 1992, pp. 329-338.

(C) Printed in India.

\title{
Application of the Linear Prediction filters in equatorial electrojet studies
}

\author{
G K RANGARAJAN \\ Indian Institute of Geomagnetism, Colaba, Bombay 400005 , India \\ MS received 24 January 1992; revised 27 July 1992
}

\begin{abstract}
Wiener filters are derived from the horizontal field data of two adjacent equatorial electrojet stations, Adis Ababa and Trivandrum from several sequences of quiet days. The time invariant property of the filter is established and the filter is applied to conditions marked by afternoon counter electrojet events. The prediction efficiency is shown to be consistently high. Possible uses of this technique in studies related to generating mechanisms of counter electrojet events and the day-to-day variability in electrojet currents are indicated. Inter-relationships and day-to-day variability of different components of the fields at the two stations are highlighted.
\end{abstract}

Keywords. Linear prediction filters; equatorial electrojet; counter electrojet

\section{Introduction}

The technique of Linear Prediction filtering was an innovation by Wiener (1942) which was adapted for application to discrete data by Levinson (1949). Much later, its usefulness in magnetospheric physics was demonstrated by Iyemori et al (1979) and Iyemori and Maeda (1980). The technique, based on least squares criteria, leads to an empirical determination of the governing linear relationship between an input function and a suitable output function. This is described in the form of a sequence of prediction filter weights which are derived from the auto-correlation coefficients of the input time series and the cross-correlation coefficients between the input and observed output. The tacit assumptions for the validity of the functional relationship are (Robinson 1967):

(i) The $X$ and $Y$ series are stationary and their errors are uncorrelated, where $X$ may be considered input and $Y$ the output series.

(ii) A linear relationship exists between $X$ and $Y$.

(iii) The linear relationship is time-invariant.

In other words, filters derived from different epochs of $X$ and $Y$ data should exhibit nearly the same response characteristic. The Wiener prediction filter or the impulse response function so derived describes the link between input and output as a function of time lag.

The optimum length of the filter can be determined from the normalized mean square error sequence as it levels off indicating no improvement in the prediction efficiency with further increase in the number of filter coefficients. We can also define 
a prediction efficiency parameter (PE) defined as

$$
\mathrm{PE}=\left(1-\sigma_{T}^{2} / \sigma_{o}^{2}\right) \times 100
$$

(Clauer et al 1983) which gives a quantitative measure of the 'goodness of fit'. $\sigma_{T}^{2}$ is the variance of the residuals and $\sigma_{o}^{2}$ that of the observed output. The impulse response proves useful in understanding the physical mechanism involved in the interaction processes governing the causative (input) and the resultant (output) parameters. An excellent review of the application of the technique in studies of solar wind-magnetosphere coupling is given by Clauer (1986). McPherron et al (1986) have shown the effectiveness of the method in real time prediction of geomagnetic activity while Fay et al (1986) have applied the Wiener technique for the Dst index and polar cap potentials.

These and other similar studies have used parameters of the solar wind and interplanetary magnetic fields as input and geomagnetic activity indices $\mathrm{AE}, \mathrm{AL}, \mathrm{Am}$ or Dst as outputs to derive the linear relationship. Instead of looking at such 'cause-andeffect' situations, we attempt to use the technique to predict the equatorial magnetic field variations at a station to the west from the data of a station to the east. It is stressed that the geomagnetic field at both the stations chosen are basically outputs of currents over the dip equator and need not be considered as 'cause-and-effect'. However, if the technique is shown to be successful, then the predicted variations can be compared with observed variations on a day-to-day basis to look for the specific physical mechanisms when the relationship fails. It can also be used to fill data gaps of the station to west from the computed values using observations at the station to the east. If the station separation is too large, the technique may not be successful as the spatial extent of correlated variations is rather restricted (Schlapp and Mann 1983). Our choice of the two equatorial electrojet stations are Adis Ababa $\left(9^{\circ} \mathrm{N} 39^{\circ} \mathrm{E}\right)$ and Trivandrum $\left(8.5^{\circ} \mathrm{N} 77^{\circ} \mathrm{E}\right)$. If there were an additional station in close proximity to Adis Ababa, the entire procedure could have been checked with the data from the third station. Unfortunately there is no possibility for this in the present case.

\section{Data selection and analysis}

The data used in the analysis are the mean hourly values of horizontal intensity at Trivandrum treated as input and that at Adis Ababa as output. For the Wiener technique to be applicable, the basic assumption that the filter is time invariant must be established. In order to ensure this, we chose our data sets to cover different phases of solar activity and different seasons. Five samples for each season in different years were identified when the index Ap of magnetic activity on 6 consecutive quiet days was $<7$ indicative of the fact that the magnetospheric conditions were quiet. The computer programme, given by Robinson (1967), was used for deriving the impulse response function in each case, after verification with his test data. The filter lengths were varied between 20 and 60 and a choice of 50 was finally adapted, as the prediction efficiency levels off in the vicinity of this length and deteriorates somewhat beyond.

As we are not considering a system where the input is a causative factor and the output is the result but one in which the two share, perhaps, the same energy source, the concept of coherence functions could also be effectively used. This alternate 
Table 1. List of 15 sequences of quiet days in different years, divided according to seasons and the corresponding daily mean Ap values.

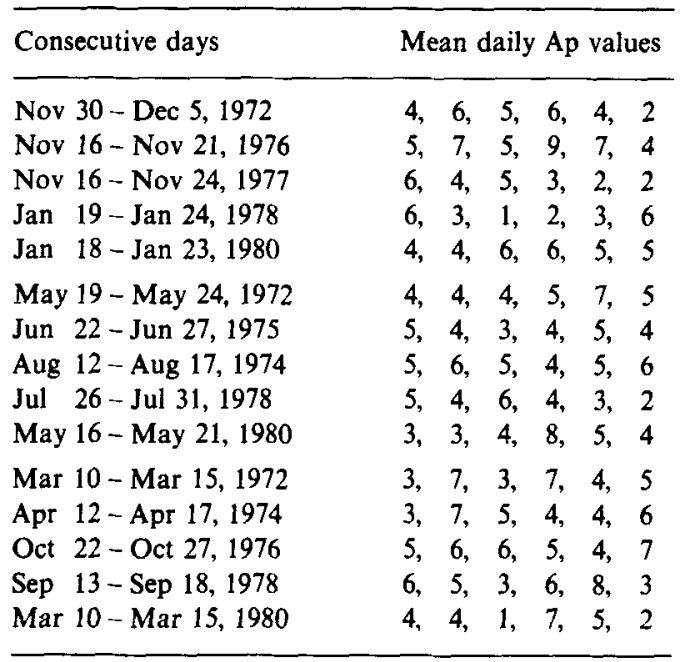

approach could be carried out through cross spectral analysis and computing the coherence and phase difference as a function of frequency (Robinson 1967). However, the Linear Prediction technique derives the impulse response as a function of time lag from the data base itself with the least squares criterion so that splitting into frequency bands is not essential. All oscillatory effects are clubbed together in the output series derived from the filter. Whenever necessary, one can easily complement the impulse response information with appropriate coherence functions. As an alternative to the coherence function to complement the L.P. technique as also for a better understanding of the longitudinal dependence of the features of equatorial electrojet, we computed the amplitudes and phases of the diurnal and semi-diurnal components of the daily variation curves for each of the 90 days, at both stations and studied their day-to-day variability. These two periodicities are the most dominant ones in the quiet-day variations close to the dip equator.

\section{Results and discussion}

The impulse response functions for the 15 samples corresponding to a filter length of 50 are shown in figure 1a. The consistent common feature of the curves is the maximum between 2 and 6 hours. This is in conformity with the expected diurnal pattern at Adis Ababa which is about $40^{\circ}$ to the west of Trivandrum. The response curves also clearly indicate that the features are not dependent on the phase of the solar cycle or the three seasons, though it is well known that the quiet-day field is stronger during equinoxes and during epochs of solar maximum (Rastogi and Iyer 1976). It is, thus, clearly established that the basic assumption of time invariance of the impulse response is valid in the present context, and therefore we can consider the use of Wiener filter in the envisaged studies. 


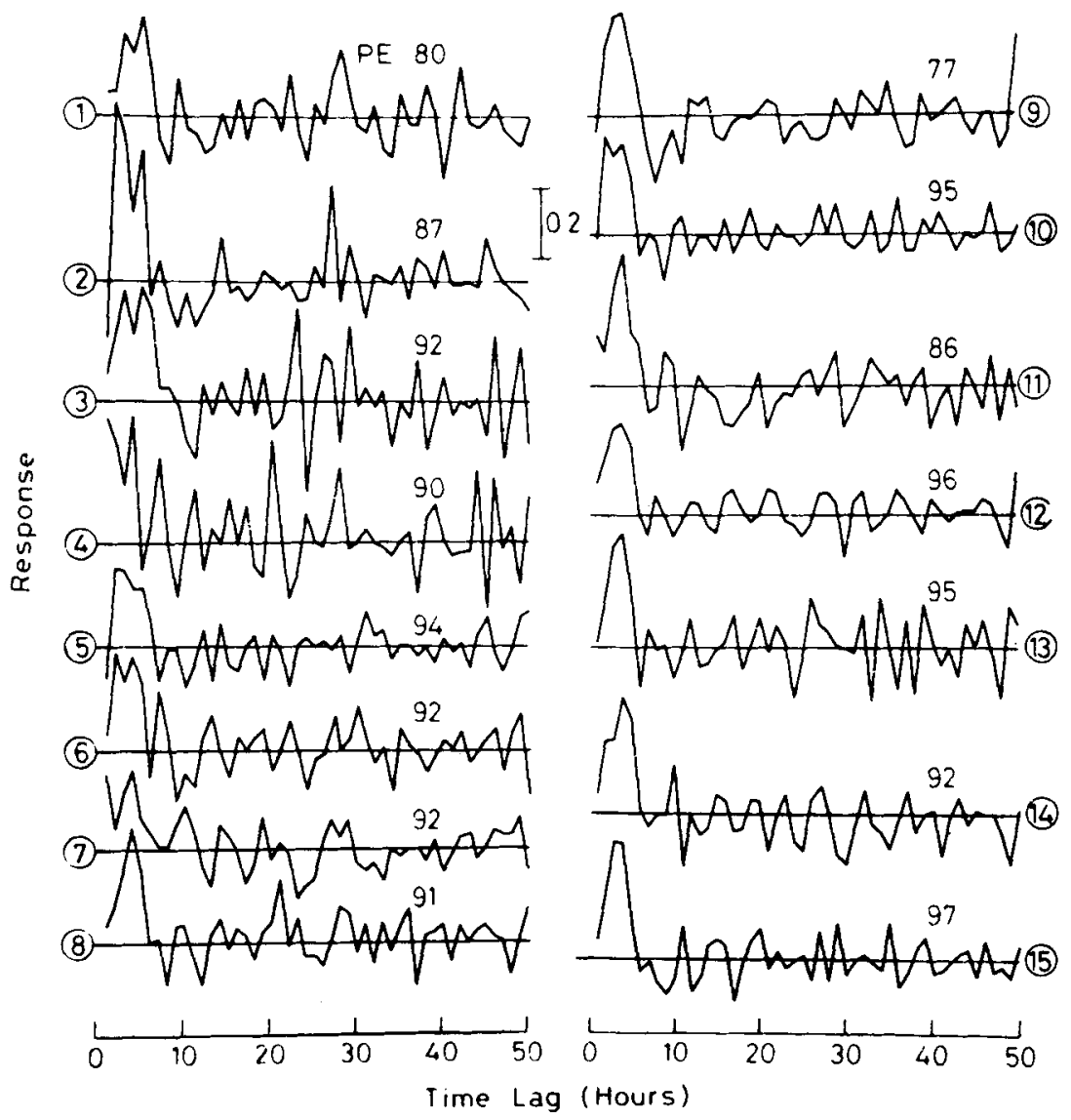

Figure 1(a). Impulse response functions relating daily variation on quiet days at Adis Ababa and Trivandrum for 15 samples listed in table 1. PE in the figure corresponds to the prediction efficiency percentage derived from variances of observed output and residuals.
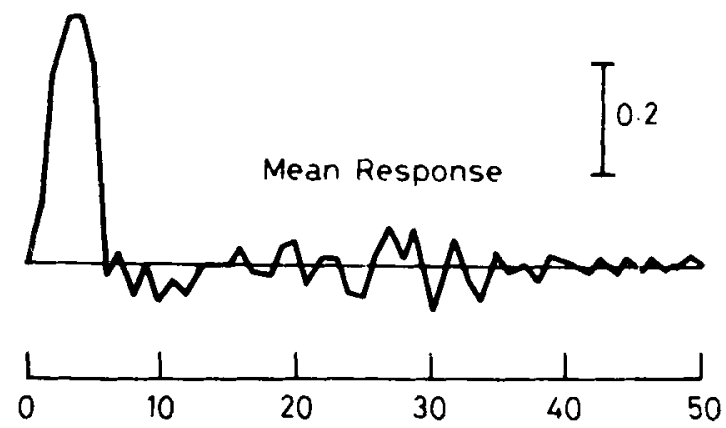

Figure 1(b). The average impulse response function derived from the 15 samples.

The prediction efficiency (PE) has a minimum percentage of 77. For 11 out of 15 cases, it exceeds $90 \%$. It is, therefore, possible to consider an average curve based on these 15 functions to derive a Wiener filter, which can then be used to infer magnetic field at Adis Ababa from Trivandrum data. The average curve derived from the 15 

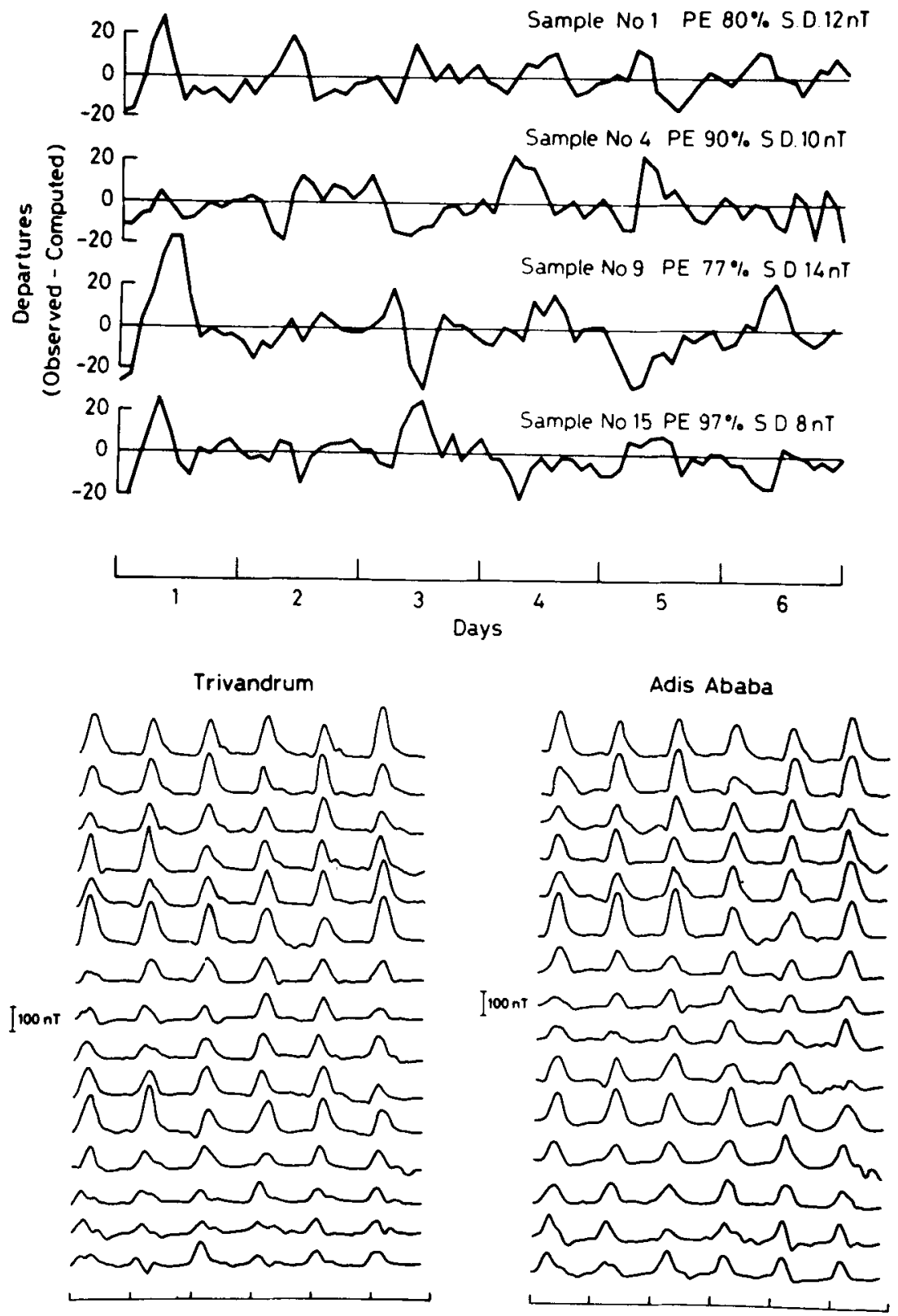

Figure 2. Mean hourly values of Horizontal intensity at Trivandrum and Adis Ababa for the 15 samples of 6 consecutive quiet days.

In the top panel are shown the residuals (computed-observed) of $\mathbf{H}$ at Adis Ababa for four typical instances. Sample number and the root mean square deviation of the residuals are indicated.

samples, is shown in figure $1 \mathrm{~b}$. As expected, the higher noise level seen in individual curves for lags beyond 10 is substantially diminished while the major feature in the earlier part is retained intact. Figure 2 shows the observed hourly values for consecutive 6 days at Trivandrum and Adis Ababa. Also shown, on the top, are the departures 
of the computed values at Adis Ababa from observations for four typical instances where the prediction efficiency varied between the minimum and maximum. It can be immediately seen that apart from the high percentage of the prediction efficiency, individual predicted hourly values do not differ much from actually observed data and that when the differences are large enough, there is no relationship to the diurnal pattern of the field variations. The root mean square of the residuals has a maximum of $14 \mathrm{nT}$, as against the diurnal range in excess of $100 \mathrm{nT}$. The high level of prediction efficiency also implies that the spatial extent of correlated equatorial changes is at least $45^{\circ}$ wide $(\sim 3$ hours).

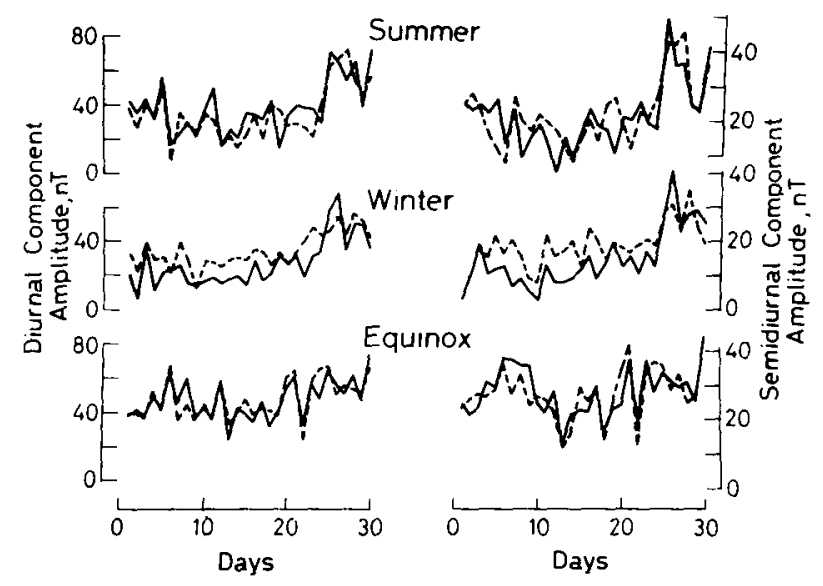

Figure 3(a). Day-to-day variability in the amplitude.

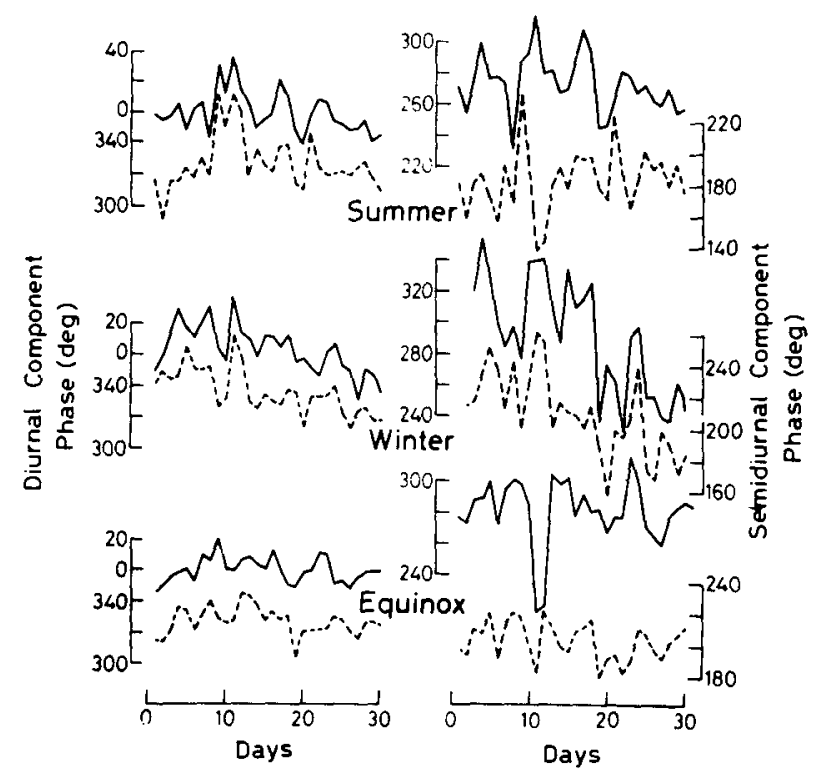

Figure 3(b). Day-to-day variability in the phase of the diurnal and semi-diurnal components for the three seasons, for the two stations Trivandrum and Adis Ababa. 


\section{Inter-relationship during counter electrojet events}

It is well known that the equatorial horizontal field is often marked by afternoon depression in the field. These are termed Counter Electrojet events and their morphology have been studied in great detail (Rastogi 1974). An objectively derived index of counter electrojet strength in the Indian zone by Bhargava et al (1983) categorised these into four classes: from index 1 for feeble depression below nighttime reference level to 4 for strong events with magnitudes of depression in excess of $40 \mathrm{nT}$. They reported that CEJ is not uncommon and that more than $50 \%$ of the days could be considered as days with afternoon CEJ event with index between 1 and 4 .

The longitudinal extent of the CEJ events is not clearly known. We attempt to find out if the linear prediction filters derived earlier can also be applied to the data when the days are marked with CEJ. For this purpose we identified 10 sequences of 6 days each with the index of CEJ between 1 and 4 with as few ones as possible from the compilation of Bhargava et al (1983). The average prediction filter derived from the earlier analysis was used to derive the predicted hourly values of Adis Ababa

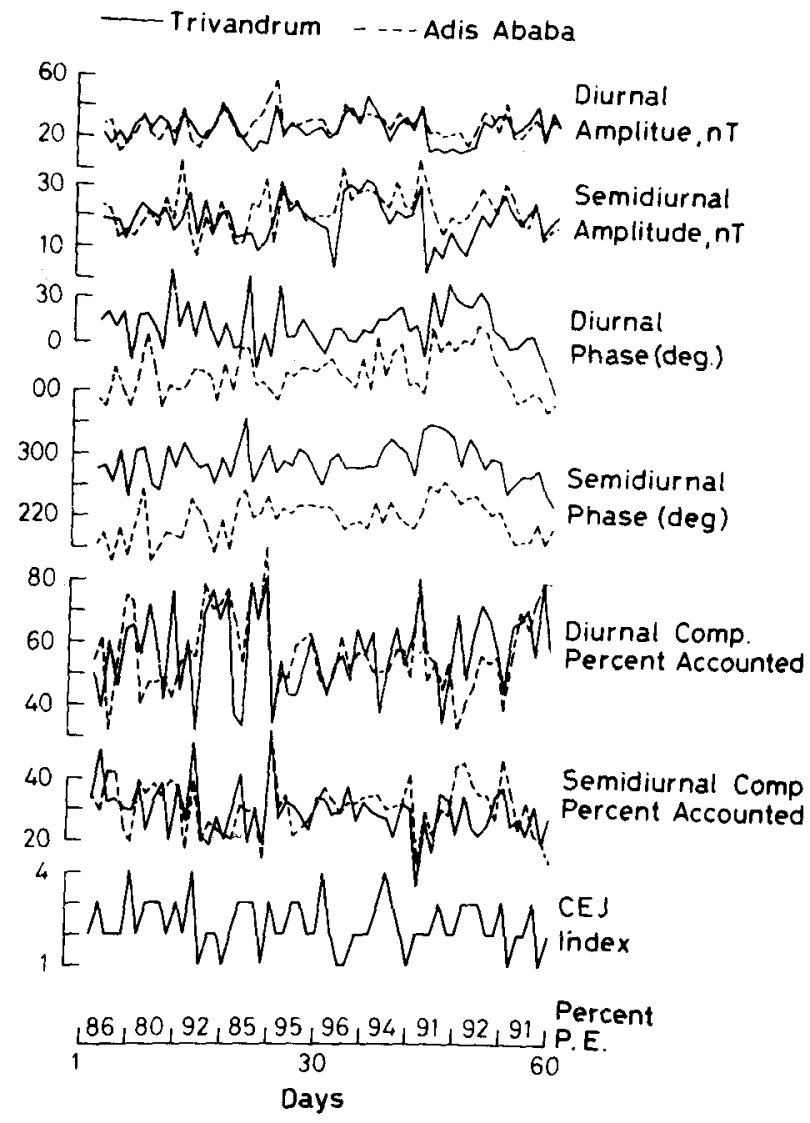

Figure 4. Prediction efficiency (PE) when the Linear filter is applied to Trivandrum $\mathbf{H}$ data to derive Adis Ababa data during periods marked by Counter Electrojet (CEJ) events. The day-to-day variability in the amplitudes and phases as well as the percentage of variance accounted for by them for all the days of CEJ are also shown. 
and the prediction efficiency was computed. These values are again consistently greater than $90 \%$ with the least value of $80 \%$ as shown in figure 4 . It is, thus, clearly demonstrated that even when the normal diurnal pattern is modified by significant afternoon depression of the field, Wiener technique is still applicable to derive the diurnal pattern of $\mathrm{H}$ field at a station to the west from the data of the station to the east.

Results of harmonic analysis for individual days are also plotted in figure 4. In addition to the amplitudes and phases of the 24 and 12 hour components we also computed the percentage of the total variance accounted for by the two dominant terms. It is well known that the semi-diurnal component gets significantly enhanced during periods of afternoon counter electrojet events (Bhargava and Sastri 1977; Somayajulu 1988). These parameters depicted in figure 4 are subjected to regression analysis and results are included in table 2 . The day-to-day variability in phase of the diurnal and semi-diurnal components is fairly similar at both stations as seen from the moderate values of $\mathrm{CC}$. The amplitudes, on the other hand, appear to be less well correlated. From these curves, it may be inferred that prediction efficiency using such pairs of stations could be significantly large when the diurnal patterns are similar in shape indicative of similarity in phase even if the amplitudes are different.

From an examination of magnetograms for 1964-65 at Adis Ababa and Kodaikanal, Rastogi (1973) suggested that the afternoon CEJ can be fairly localized and may not be concurrent at two stations even if they are separated only by 2 or 3 hours in longitude. Rao and Rao (1963) found that the significant depressions in $\mathrm{H}$ magnetogram of Adis Ababa found by Gouin (1962) on January 3 and 5, 1962 were significantly absent in the records at the Indian stations Trivandrum and Kodaikanal. These individual events clearly show that there could be significant interference in the current systems responsible for the CEJ over a short duration of time on some occasions. The mechanism(s) responsible for these are yet to be clearly understood.

The technique proposed here gives an opportunity to firstly catalogue the occasions when the predicted variations of Adis Ababa $\mathrm{H}$ differs significantly from the observations and then compare the ionospheric and wind parameters (if available) on these occasions at both the locations to isolate the causes. The statistics would also be useful in validating theories for the counter-electrojet phenomena and the day-to-day variability in the $\mathrm{Sq}$ and electrojet currents.

\section{Conclusions}

Wiener's Linear Prediction technique to derive the impulse response functions can be successfully applied to the data from a pair of equatorial electrojet stations so that variations of the field at the station in the western longitude can be derived from the data of a station located to the east. This, to our mind, is a new application in contrast to the demonstrated usage in solar wind-magnetosphere coupling studies where physical mechanisms were being sought to explain the observed changes in geomagnetic activity and has potential uses in understanding the causative mechanisms for equatorial CEJ, day-to-day variability of $\mathrm{Sq}$ and $\mathrm{EJ}$ etc. The impulse response function is clearly shown to be independent of season and solar activity thus satisfying the most rigorous criterion of the technique. Some characteristic features of the equatorial electrojet like the longitudinal extent of correlated changes, the interrelationship of the day-to-day variability can be verified by adopting this technique 
for suitable pairs of stations with increasing longitudinal separation. Days when the predicted diurnal pattern does not match closely with the observed variation could be examined in great detail for other influencing parameters like global Sq, equatorial electric field, F-region contributions, IMF or solar wind variability.

\section{Acknowledgements}

The author thanks the referees for useful comments which helped in improving the quality of the manuscript.

\section{References}

Bhargava B N and Sastri N S 1977 A comparison of days with and without occurrence of counter electrojet afternoon events in the Indian region; Ann. Geophys. 33 329-332

Bhargava B N, Arora B R and Sastri N S 1983 Indices of equatorial electrojet and counter electrojet in the Indian region: Evolution of the indices and their authenticity; Proc. Indian Acad. Sci. Earth Planet. Sci. $9245-56$

Clauer C R 1986 The technique of linear prediction filters applied to studies of solar wind-magnetosphere coupling in "Solar wind-Magnetosphere Coupling", (eds) Y Kamide and J A Slavin; Terra Pub, Tokyo, Japan pp. 39-58

Clauer C R, McPherron R L and Searls C 1983 Solar wind control of low latitude asymmetric field; $J$. Geophys. Res. 88 2123-2130

Fay R A, Garrity C R, McPherron R L and Bargatze L F 1986 Prediction filters for the Dst index and the polar cap potential in "Solar wind-Magnetosphere Coupling", (eds) Y Kamide and J A Slavin; Terra Pub. Tokyo. Japan pp. 111-118

Gouin P 1962 Reversal of the magnetic daily variation at Adis Ababa; Nature (London) 193 1145-1146

Iyemori $T$, Maeda $H$ and Kamei $T 1979$ Impulse response of geomagnetic indices to interplanetary magnetic field; J. Geomagn. Geoelectr. 31 1-9

Iyemori T and Maeda $\mathrm{H} 1980$ Prediction of geomagnetic activities from solar wind parameters based on the linear prediction theory; Solar Terr. Prediction Proceedings, US Dept. of Commerce Boulder CO, USA, Vol. 4 A 1

Levinson N 1949 The Wiener R M S (Root Mean Square) error criterion in filter design and prediction in Appendix B. N. Wiener's book reprint

McPherron R L, Baker B D N and Bargatze L F 1986 Linear filters as a method of real time prediction of geomagnetic activity in "Solar wind-Magnetosphere Coupling", (eds) Y Kamide and J A Slavin; Terra Pub, Tokyo 85-96

Rao K N and Raja Rao K S 1963 Quiet day magnetic variations near the magnetic equator; Nature (London) $200460-461$

Rastogi R G 1973 Counter equatorial electrojet currents in Indian zone Planet; Space Sci. 21 1355-1365

Rastogi R G 1974 Westward equatorial electrojet during daytime hours; J. Geophys. Res. 79 1503-1512

Rastogi R G and lyer K N 1976 Quiet day variation of geomagnetic H field at low latitudes; J. Geomagn. Geoelectr. $28461-479$

Robinson E A 1967 Multi-channel time series analysis with digital computer programs Holden Day, San Francisco, 232-259

Somayajulu V V 1988 Behaviour of harmonic components of the geomagnetic field during counter electrojet events; J. Geomagn. Geoelectr. 40 111-130

Schlapp D H and Mann R J 1983 The spatial scale of correlation of the day-to-day variability of Sq Geophys.; J. R. Astron. Soc. 73 671-673

Wiener N 1942 Extrapolation interpolation and smoothing of stationary time series with engineering applications. (Cambridge Mass: MIT Press) 